RESEARCH HIGHLIGHT

\title{
Kappa-opioid antagonists as stress resilience medications for the treatment of alcohol use disorders
}

\author{
Charles Chavkin (iD) \\ Neuropsychopharmacology (2018) 43:1803-1804; https://doi.org/10.1038/s41386-018-0046-4
}

Extensive preclinical data support the proposal that kappa-opioid receptor antagonists may have therapeutic benefit for the treatment of mood disorders and drug addiction by promoting stress resilience [1]. People who are vulnerable to depression, anxiety, or addiction can become hypersensitive to stressful experience, which can act as a trigger for a recurrence of a mood disorder or substance use episode. The neuronal processes underlying these responses have been extensively studied in animal models where stress exposure can cause the release of stress neuropeptides including the endogenous dynorphin opioids in brain, which activate kappaopioid receptors. Kappa receptor activation in animals and humans produces anxiety-like, dysphoric, aversive, and drug-seeking behavioral responses. Antagonists that block kappa-opioid receptors can reduce these stress responses and thereby have antidepressant and anti-addictive activity in animal models. For example, kappa antagonists block stress-potentiation of drug reward, block drugseeking in substance-dependent animals, and block stress-induced reinstatement of drug self-administration. These beneficial effects of kappa antagonists are evident in behavioral assays using a wide range of addictive substances: cocaine, heroin, nicotine, ethanol, and methamphetamine [2]. In addition, kappa antagonists are effective in the preclinical behavioral assays predictive of antidepressant efficacy in humans [1]. Thus, kappa antagonists could theoretically have broad clinical utility, and the recognition of this opportunity has stimulated a new wave of drug development.

Clinical trials with buprenorphine (a nonselective drug having kappa receptor antagonist activity) support this concept [3], and several pharmaceutical companies have been actively developing selective kappa-opioid receptor antagonists for use in human studies. It is important to note that there are two different types of kappa antagonists: the initially introduced compounds (e.g., norbinalorphimine and JDTic) are highly selective KOR antagonists but can inactivate the kappa receptor for weeks ("receptorinactivating antagonists"). The mechanism for long-lasting kappa receptor inactivation by this type of antagonist was recently described and involves the activation of a novel signaling pathway initiated by c-Jun Kinase [4]. The kappa receptor signaling complex undergoes as a structural change after antagonist binding that permanently inactivates signaling, even after drug dissociation. In contrast, short-acting kappa selective compounds have been synthesized that act as competitive inhibitors of agonist action ("conventional antagonists"). The short-acting kappa antagonist developed by Eli Lilly [(S)-3-fluoro-4-(4-((2-(3,5dimethylphenyl)pyrrolidin-1yl)methyl)-phenoxy)benzamide], known as LY-2456302 or CERC-501, is the farthest along in clinical development, currently by Janssen Pharmaceuticals.
Whether a long-acting, receptor-inactivating kappa antagonist would be a better therapeutic than a short-acting, conventional antagonist is not yet clear. Drugs having long durations of action would produce more stable responses, which has theoretical advantages. But the ability to quickly adjust the response by changing the dose of a short-acting antagonist would allow more efficient treatment optimization. Most of the preclinical studies todate have used the long-acting compounds, norbinaltorphime, or JDTic, at doses that completely inactivate kappa receptor signaling to establish the therapeutic potential. A limited number of studies using the conventional kappa antagonist buprenorphine have demonstrated its effectiveness, but buprenorphine lacks receptor selectivity, which prevents the attribution of its antidepressant effects solely to kappa antagonism. Similarly, high-doses of naltrexone are effective, but non-selectively inhibit mu, delta, and kappa-opioid receptors. The safety of the different forms of kappa antagonist is also a concern. Initial results suggest that the long-acting drug JDTic produced adverse cardiovascular effects in human subjects, whereas the short-acting antagonist CERC-501 did not produce adverse effects in Phase II human trials (https:// www.dddmag.com/article/2017/08/j-j-adds-new-depression-drugportfolio). Whether long-acting kappa antagonists produce cardiovascular toxicity as a class effect is not known, and additional studies are required to determine whether conventional or inactivating kappa receptor antagonists are safer and more effective. Nevertheless, drug development is currently focused on the conventional antagonists because of the perception that these would be more 'drug-like', and the preclinical study of CERC-501 by Domi et al. [5], published in this issue of Neuropsychopharmacology is an important advance in this translational effort.

CERC-501 is a selective, orally available and centrally penetrant kappa antagonist that has been found to be safe in Phase 1 human trials. Markus Heilig and colleagues recently tested CERC501 in a battery of preclinical assays of alcohol addiction treatment. Male Wistar rats receiving an acute administration of ethanol show anxiety-like responses in the elevated plus maze during the withdrawal phase, and oral administration of CERC-501 blocked this response. Rats trained to lever press for ethanol reinstated extinguished self-administration when stressed by footshock, and CERC-501 blocked reinstatement. In contrast, CERC-501 did not affect cue-induced reinstatement. Some rats given intermittent or continuous access to alcohol escalated their consumption, whereas some did not. CERC-501 suppressed alcohol self-administration in the escalators but did not affect consumption in the rats that that did not escalate.

'Department of Pharmacology, University of Washington, Seattle, WA 98195, USA

Correspondence: Charles Chavkin (cchavkin@u.washington.edu)

Received: 10 February 2018 Revised: 27 February 2018 Accepted: 3 March 2018

Published online: 27 March 2018 
These results are very encouraging and support the concept that a conventional, short-acting, and selective kappa receptor antagonist can be a safe and effective medication. Key questions remain however. We still know too little about how the dynorphins function in human brain. Brain imaging studies are required to determine if dynorphin tone is elevated during periods of clinical depression; during periods of abstinence from nicotine, alcohol or opiates; or during periods of psychotic confusion. Kappa antagonists are unlikely to beneficial unless elevated dynorphin tone is responsible for the adverse responses or if they can block constitutive activity of KOR initiated by dynorphin action [6]. Recognizing that mood disorders and substance use disorders are complex and multifactorial, kappa antagonists are not suggested as a cure. Instead, we predict that medications that promote stress resilience may become useful adjuncts in their clinical management.

\section{ADDITIONAL INFORMATION}

Competing interests: The author declares no competing interests.

Publisher's note: Springer Nature remains neutral with regard to jurisdictional claims in published maps and institutional affiliations.

\section{REFERENCES}

1. Carroll Fl, Carlezon WA Jr. Development of $\mathrm{k}$ opioid receptor antagonists. J Med Chem. 2013;56:2178-95.

2. Chavkin C, Koob GF. Dynorphin, dysphoria, and dependence: The stress of addiction. Neuropsychopharmacology. 2016;41:373-4.

3. Fava M, Memisoglu A, Thase ME, Bodkin JA, Trivedi MH, de Somer M, Du Y, LeighPemberton R, DiPetrillo L, Silverman B, Ehrich E. Opioid modulation with buprenorphine/samidorphan as adjunctive treatment for inadequate response to antidepressants: a randomized double-blind placebo-controlled trial. Am J Psychiatry. 2016;173:499-508.

4. Schattauer SS, Land BB, Reichard KL, Abraham AD, Burgeno LM, Kuhar JR, Phillips PEM, Ong SE, Chavkin C. Peroxiredoxin 6 mediates Gai protein-coupled receptor inactivation by cJun kinase. Nat Commun. 2017;8:743.

5. Domi E, Barbier E, Augier E, Augier G, Gehlert D, Barchiesi R, Thorsell A, Holm L, Heilig M. Preclinical evaluation of the kappa-opioid receptor antagonist CERC-501 as a candidate therapeutic for alcohol use disorders. Neuropsychopharmacology. 2018; https://doi.org/10.1038/s41386-018-0015-y.

6. Polter AM, Barcomb K, Chen RW, Dingess PM, Graziane NM, Brown TE, Kauer JA. Constitutive activation of kappa opioid receptors at ventral tegmental area inhibitory synapses following acute stress. eLife. 2017;6:e23785. 